\title{
Nitric oxide in children with persistent asthma
}

\author{
Óxido nítrico em criança com asma persistente \\ Nulma S. Jentzsch ${ }^{1}$, Muriel le Bourgeois ${ }^{2}$, Jacques de Blic ${ }^{3}$, \\ Pierre Scheinmann ${ }^{4}$, Serge Waernessyckle ${ }^{5}$, Paulo A. M. Camargos 6
}

\section{Resumo}

Objetivo: Verificar as diferenças nos valores da fração exalada de óxido nítrico (FeNO) em asmáticos atópicos e não-atópicos em uso de tratamento antiinflamatório e comparar a FeNO com a função pulmonar.

Métodos: Estudo transversal com 45 asmáticos persistentes moderados e graves, de 6 a 17 anos, selecionados consecutivamente, em uso de medicação antiinflamatória há pelo menos 1 ano. Os pacientes foram divididos em dois grupos: atópicos, isto é, com testes cutâneos positivos, e não-atópicos. As avaliações clínico-funcionais e a mensuração da FeNO foram realizadas concomitantemente.

Resultados: Houve predomínio do sexo masculino (62,5\%), sendo que cerca de $85 \%$ pertenciam à faixa etária de 6 até 13 anos (média, 10,4 anos). Não foi encontrada, nos dois grupos, significância estatística para a presença de sintomas associados à asma $(p=0,07)$, rinite alérgica $(p=0,17)$, alergia alimentar $(p=0,09)$, necessidade de corticóide sistêmico $(p=0,10)$, antileucotrieno $(p=0,20)$ e antihistamínico $(p=0,70)$, nem para os três parâmetros usados para avaliar a função pulmonar $\left(\mathrm{VEF}_{1}, \mathrm{VEF}_{1} / \mathrm{CVF}\right.$ e $\left.\mathrm{FEF}_{25-75 \%,} \mathrm{p} \geq 0,14\right)$. A freqüência de eczema $(p<0,005)$ e a FeNO $(p<0,001)$ foram mais elevadas entre os atópicos.

Conclusão: Os resultados sugerem que, entre atópicos, a estabilidade clínica e funcional da asma não reflete, necessariamente, o efetivo controle do processo inflamatório, e que haja, talvez, maior chance de recidiva após a suspensão da medicação anti-inflamatória.

J Pediatr (Rio J). 2006;82(3):193-6: Asma, atopia, fração exalada de óxido nítrico.

\section{Abstract}

Objective: To assess the difference in exhaled nitric oxide levels in atopic and nonatopic asthmatic patients treated with antiinflammatory drugs, and to compare exhaled nitric oxide measurement with lung function tests.

Methods: Cross-sectional study with 45 consecutively selected patients with moderate and severe persistent asthma, aged between 6 and 17 years, and treated with anti-inflammatory drugs for at least 1 year. The patients were split into two groups: atopic ones (with positive skin tests) and nonatopic ones. The clinical and functional assessments and the measurement of exhaled nitric oxide were carried out concomitantly.

Results: There was a male predominance $(62.5 \%)$, with an age range between 6 and 13 years (mean of 10.4 years) in $85 \%$ of the patients. Neither the symptoms associated with asthma $(p=0.07)$, allergic rhinitis $(p=0.17)$, food allergy $(p=0.09)$, necessity of systemic corticosteroids $(p=0.10)$, antileukotrienes $(p=0.20)$ and antihistamines $(p=0.70)$, nor the three parameters used to assess lung function ( $\mathrm{FEV}_{1}$,

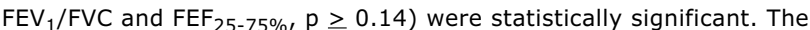
frequency of eczema $(p<0.005)$ and exhaled nitric oxide levels $(p<0.001)$ were higher among atopic patients.

Conclusion: Results suggest that clinical and functional stability of asthma among atopic patients does not necessarily reflect an efficient control over the inflammatory process and a higher probability for recurrence after discontinuation of anti-inflammatory therapy.

J Pediatr (Rio J). 2006;82(3):193-6: Asthma, atopy, exhaled nitric oxide.

\section{Introdução}

O processo inflamatório crônico das vias aéreas ocupa um lugar de destaque na asma, mas nenhum marcador dessa inflamação é rotineiramente usado na prática pediátrica. O óxido nítrico (NO) é considerado um marcador dessa inflamação. A concentração da fração exalada do óxido nítrico (FeNO) é um índice útil, podendo ser medida instantaneamente (on-line) por métodos sensíveis, práticos, fáceis, não-invasivos, os quais fornecem resultados imediatos, confiáveis e estandardizados ${ }^{1,2}$. A análise da FeNO pode auxiliar tanto no diagnóstico quanto no monitoramento da resposta à medicação antiinflamatória, já que se apresenta aumentada nos pacientes virgens de tratamento e, em razão da ampla variabilidade clínico-funcional dessa afecção, os ajustamentos terapêuticos são freqüentemente necessários ${ }^{3-7}$.

A utilização da FeNO pode contribuir para a detecção de alterações sub-clínicas, insuficiência do tratamento antiin- 
flamatório e adesão ao tratamento, além de identificar pacientes sob risco de exacerbação da asma ${ }^{8}$. Os corticóides inalatórios (CI) ou sistêmicos reduzem a FeNO 9,10 . Assim, a FeNO pode ser empregada para monitorar e quantificar a inflamação e, por isso, é útil no controle individual e, em especial, no manejo da dose do CI.

$O$ presente estudo tem como objetivo mensurar o perfil clínico-funcional e a FeNO em crianças e adolescentes, atópicos e não-atópicos, com asma persistente.

\section{Métodos}

\section{População e local de realização do estudo}

Trata-se de um estudo transversal, desenvolvido no Serviço de Pneumologia Pediátrica e Alergologia do Hospital Necker-Enfants Malades (Paris, França), no qual foram incluídas 45 crianças asmáticas selecionadas consecutivamente.

\section{Critérios de inclusão e exclusão}

Foram admitidos pacientes com asma persistente moderada e grave, segundo critérios do Global Initiative for Asthma ${ }^{11}$, em uso de corticoterapia inalatória há pelo menos 12 meses. As crianças com asma intermitente ou persistente leve que não receberam $\mathrm{CI}$ e que apresentavam outras afecções subjacentes foram excluídas.

\section{Grupos estudados}

As crianças e adolescentes participantes do estudo foram classificados em atópicos e não-atópicos, sendo que a atopia foi definida pela presença de testes cutâneos positivos.

\section{Verificação da medida da fração exalada de óxido nítrico}

A medida da FeNO foi realizada on-line, com equipamento Endono ${ }^{\circledR}$ (Seres, Aix-en-Provence, França), com a criança assentada e confortavelmente instalada, sem atividade física prévia ao exame e sem uso de qualquer medicação no dia do exame. A medida da FeNO é feita através da quimiluminescência, método que consiste na contagem do número de fótons emitidos no retorno ao estado estável das moléculas de NO produzidas pela reação do NO em presença de excesso de ozônio $\left(\mathrm{O}_{3}\right)$; o sinal luminoso medido é proporcional à concentração de NO.

O NO foi medido durante uma expiração prolongada, de pelo menos 6 segundos, a fim de obter um plateau de pelo menos 3 segundos, a um fluxo de $50 \mathrm{ml} / \mathrm{s}^{12}$. Um plateau de 2 segundos em crianças menores de 12 anos reduz a confiabilidade do exame ${ }^{13}$.

Os aparelhos existentes para fazer esse exame detectam o NO de origem broncopulmonar nos valores mínimos de uma parte por bilhão (ppb) ${ }^{14}$. O ponto de corte tomado no presente estudo para o limite superior da normalidade foi de $10 \mathrm{ppb}$, de acordo com a especificidade do aparelho Endono $^{\circledR}$ e segundo Franklin ${ }^{15}$, além de ser o valor de referência adotado no serviço onde foi realizada a pesquisa.
As concentrações da FeNO variam de acordo com os diferentes equipamentos e fabricantes, situando-se entre 10 a 20 ppb. Porém, em crianças e adolescentes hígidos, a literatura registra valores em torno de $10 \mathrm{ppb}$, variando de $7,2 \mathrm{ppb}(6,4-8,0 \mathrm{ppb})^{15}$ a $8,7 \mathrm{ppb}(8,1-9,2 \mathrm{ppb})^{16}$. Essas concentrações são elevadas nos asmáticos, com taxas 1,6 a 4,4 vezes maior que nos indivíduos hígidos ${ }^{17}$. Durante o tratamento com CI ou sistêmico, há uma queda, e mesmo normalização, desses valores.

\section{Aspectos estatísticos}

A análise incluiu os cálculos de distribuição de freqüência, médias, qui-quadrado, teste exato de Fisher e teste $t$ de Student.

\section{Resultados}

Entre as 45 crianças e adolescentes, 24 delas (53,3\%) eram atópicas e $21(46,6 \%)$, não-atópicas. A seguir, são apresentadas as características da população estudada (Tabela 1).

Houve predomínio do sexo masculino $(62,5 \%)$, sendo que cerca de $85 \%$ tinham entre 6 e 13 anos. A classificação da gravidade da asma foi semelhante nos dois grupos $(p=1,0)$, e o grupo atópico teve maior proporção de eczema ( $p=0,005)$. Não houve significância estatística para a presença de sintomas $(p=0,07)$, rinite alérgica $(p=0,17)$, alergia alimentar $(p=0,09)$, necessidade maior de uso corticóide sistêmico $(p=0,10)$, antileucotrieno $(p=0,20)$ e anti-histamínico $(p=0,70)$.

Todos os pacientes estavam em uso de $\mathrm{CI}$; no primeiro grupo, a dose média de CI foi de $396 \mu \mathrm{g}( \pm \mathrm{DP} 78,0)$ e, no outro grupo, de 264,3 $\mathrm{gg}$ ( \pm DP 80,0$)$ ( $p=0,002$ ).

$\mathrm{Na}$ Tabela 2, encontram-se os resultados dos testes de função pulmonar, cujos parâmetros foram expressos em porcentagem do valor previsto e da mensuração da FeNO.

Embora não tenha sido encontrada significância estatística para os três parâmetros usados para avaliar a função pulmonar $\left(\mathrm{VEF}_{1}, \mathrm{VEF}_{1} / \mathrm{CVF}\right.$ e $\left.\mathrm{FEF}_{25-75 \%}\right)$, quando se mediu a FeNO, observou-se uma diferença $(16,7 \mathrm{ppb}$ e 5,3 ppb) estatisticamente significativa entre os dois grupos ( $p<0,01)$, sendo que o grupo de asmáticos atópicos apresentou uma concentração média da FeNO 3,1 vezes superior, resultados que revelam a persistência da atividade inflamatória a despeito do uso de medicação antiinflamatória.

Sete pacientes estavam em uso de corticóide sistêmico (ver Tabela 1), seis crianças atópicas e uma não-atópica. Mesmo sendo uma droga recomendada na asma persistente grave, a taxa do FeNO entre esses atópicos foi expressivamente mais elevada (respectivamente, $16,5 \mathrm{ppb}, 64,0 \mathrm{ppb}$, 31,5 ppb, 16,0 ppb 16,0 ppb e 16,0 ppb, média de 26,6 ppb) que aquela obtida para o único paciente sem atopia $(5,3$ ppb, $\mathrm{p}<0,001)$.

\section{Discussão}

A média dos valores da FeNO (5,3 ppb) encontrada no grupo não-atópico, após tratamento com $\mathrm{CI}$, antileucotrie- 
no e anti-histamínico está de acordo com os valores de referência ${ }^{15-17}$.

Não há um consenso sobre a presença de sintomas ser maior no asmático atópico. No presente estudo, não foi encontrada diferença estatisticamente significativa nos dois grupos, porém alguns autores relatam essa significância ${ }^{18,19}$.
O presente estudo revelou que, apesar dos dois grupos estarem em tratamento da asma com CI, e/ou antileucotrieno, e/ou anti-histamínico e/ou corticóide sistêmico, as crianças asmáticas atópicas tiveram a FeNO significativamente mais elevada, quando comparadas com crianças asmáticas não-atópicas $(\mathrm{p}<0,001)$, fato que está de acordo com estudos sobre o comportamento da FeNO na atopia.

Tabela 1 - Características descritivas da população estudada

\begin{tabular}{|c|c|c|c|c|c|}
\hline \multirow[t]{2}{*}{ Variável } & \multicolumn{2}{|c|}{$\begin{array}{l}\text { Asmáticos atópicos } \\
\qquad(n=24)\end{array}$} & \multicolumn{2}{|c|}{$\begin{array}{l}\text { Asmáticos não-atópicos } \\
\qquad(\mathrm{n}=\mathbf{2 1})\end{array}$} & \multirow[t]{2}{*}{$\mathbf{p}$} \\
\hline & $\mathbf{n}$ & $\%$ & $\mathbf{n}$ & $\%$ & \\
\hline \multicolumn{6}{|l|}{ Sexo } \\
\hline Masculino & 15 & 62,5 & 14 & 66,7 & \\
\hline Feminino & 9 & 37,5 & 7 & 33,3 & 0,98 \\
\hline \multicolumn{6}{|l|}{ Idade (anos) } \\
\hline $6-10$ & 10 & 41,7 & 13 & 61,9 & \\
\hline $11-13$ & 10 & 41,7 & 6 & 28,6 & \\
\hline $14-17$ & 4 & 16,6 & 2 & 9,5 & 0,29 \\
\hline \multicolumn{6}{|c|}{ Gravidade da asma } \\
\hline Moderada & 2 & 8,3 & 2 & 9,6 & \\
\hline Grave & 22 & 91,7 & 19 & 90,4 & 1,0 \\
\hline Sintomas & & & & & 0,07 \\
\hline Presente & 13 & 54,2 & 5 & 23,8 & \\
\hline Ausente & 11 & 45,8 & 16 & 76,2 & \\
\hline \multicolumn{6}{|l|}{ Rinite alérgica } \\
\hline Presente & 8 & 33,3 & 3 & 14,3 & \\
\hline Ausente & 16 & 66,7 & 18 & 85,7 & 0,17 \\
\hline \multicolumn{6}{|l|}{ Eczema } \\
\hline Presente & 6 & 25,0 & zero & 0,0 & \\
\hline Ausente & 18 & 75,0 & 21 & 100,0 & 0,005 \\
\hline \multicolumn{6}{|c|}{ Alergia alimentar } \\
\hline Presente & 2 & 8,3 & zero & 0,0 & \\
\hline Ausente & 22 & 91,7 & 21 & 100,0 & 0,09 \\
\hline \multicolumn{6}{|c|}{$\begin{array}{l}\text { Dose correspondente de } \\
\text { beclometasona }\end{array}$} \\
\hline Até $800 \mu \mathrm{g}$ & 20 & 83,3 & 20 & 95,2 & \\
\hline$>800 \mu \mathrm{g}$ & 4 & 16,7 & 1 & 4,8 & 0,35 \\
\hline \multicolumn{6}{|c|}{ Uso de antileucotrieno } \\
\hline Sim & 9 & 37,5 & 4 & 19,0 & \\
\hline Não & 15 & 62,5 & 17 & 81,0 & 0,20 \\
\hline \multicolumn{6}{|c|}{ Uso de anti-histamínico } \\
\hline Sim & 5 & 20,8 & 3 & 14,3 & \\
\hline Não & 19 & 79,2 & 18 & 85,7 & 0,70 \\
\hline \multicolumn{6}{|c|}{ Uso de corticóide sistêmico } \\
\hline Sim & 6 & 25,0 & 1 & 4,7 & \\
\hline Não & 18 & 75,0 & 20 & 95,3 & 0,10 \\
\hline
\end{tabular}

Tabela 2 - Resultados da espirometria e da FeNO em asmáticos atópicos e não-atópicos

\begin{tabular}{lccc}
\hline & $\begin{array}{c}\text { Asmáticos atópicos } \\
(\mathbf{n = 2 4 )}\end{array}$ & $\begin{array}{c}\text { Asmáticos não-atópicos } \\
(\mathbf{n}=\mathbf{2 1})\end{array}$ & $\mathbf{p}$ \\
\hline $\mathrm{VEF}_{1}$ (\% do valor previsto) & 99,1 & 95,1 & 0,23 \\
$\mathrm{VEF}_{1}$ /CVF (\% do valor previsto) & 95,8 & 92,0 & 0,14 \\
$\mathrm{FEF}_{25}$-75 (\% do valor previsto) & 82,5 & 80,6 & 0,5 \\
FeNO (ppb) & 16,7 & 5,3 & $<0,001$ \\
\hline
\end{tabular}

$\mathrm{FEF}_{25-75}=$ fluxo expiratório forçado $25-75 \%$; FeNO = fração exalada do óxido nítrico em partes por bilhão; $V_{E F}=$ volume $_{1}$ expiratório forçado no primeiro segundo; $\mathrm{VEF}_{1} / \mathrm{CVF}=$ volume expiratório forçado no primeiro segundo/capacidade vital forçada. 
Leuppi et al.20, em trabalho com 235 crianças de 8 a 14 anos, encontrou nos atópicos FeNO de 18,6 ppb e de 12,9 ppb nos indivíduos sem atopia $(p=0,001)$. Estudo semeIhante conduzido por Broxai et al. ${ }^{21}$ com 429 crianças mostrou média geométrica da FeNO de 10 ppb nos atópicos e de 7,7 ppb em não-atópicos ( $<<0,01)$. Da mesma forma, Silvestri et al. ${ }^{22}$ avaliaram 112 crianças atópicas e nãoatópicas, com asma persistente moderada e grave e média de idade de 10,9 anos, encontrando FeNO de 23,9 ppb nos atópicos e de 7,6 ppb nos pacientes não-atópicos $(p=0,0001)$. Esses resultados sugerem que a atopia por si mesma induz a produção de diferentes populações celulares e/ou produção de diferentes citocinas, independentemente do uso de medicação antiinflamatória. Esses autores admitiram que a queda do $\mathrm{pH}$ das vias aéreas ocasiona broncoespasmo, formação de substâncias pró-inflamatórias e conversão do dióxido de nitrogênio em NO. Cabe salientar que, como no presente estudo, Silvestri et al. não encontraram correlação entre a FeNO e parâmetros pulmonares $(p>0,1)^{22}$.

Os resultados são particularmente interessantes quando se analisa a função pulmonar, cujos parâmetros não apresentaram correspondência com a FeNO, demonstrando que espirometria normal não reflete a ausência de processo inflamatório em atividade e, diante de valores normais, não é possível afirmar que um dado paciente está com a asma sob controle.

A persistência da FeNO elevada pode ser devida ao não controle da inflamação. Neste estudo, ela manteve-se nas seis crianças atópicas em uso de corticóide sistêmico (26,6 $\mathrm{ppb})$. Buchvald et al. ${ }^{23}$ levantam a hipótese de haver indivíduos que apresentam uma heterogeneidade de resposta da FeNO aos corticóides.

O tipo de estudo, transversal, é sujeito a limitações, mas pode oferecer informações para possível mudança, tanto para a redução quanto para o aumento da dose do CI na profilaxia da asma. É o que mostra o estudo de Smith et al. ${ }^{24}$, com 46 pacientes de 12 a 75 anos, que utilizou a dosagem da FeNO para ajustar a dose do CI. No final do período de observação, verificaram que esse grupo usou $45 \%$ menos CI do que no grupo controle de 48 pessoas tratadas de acordo com consensos internacionais. A dose de fluticasona foi de $370 \mu \mathrm{g}$ e $641 \mu \mathrm{g}$, respectivamente $(p=0,003)$. A taxa de exacerbações foi maior no grupo controle $(p=0,23)$, no qual a média da FeNO foi de 6,4 ppb. Os autores concluíram que o grupo controle usou uma dose muito mais alta de CI que o necessário e sugerem que a FeNO é uma alternativa lógica para ajuste do CI. Por sua vez, Pijnenburg et al. ${ }^{25}$ verificaram que alguns pacientes atópicos permanecem com níveis elevados da FeNO, mesmo em uso prolongado de $\mathrm{CI}$, e que tais níveis permaneceram aumentados, mesmo com o uso correto da técnica inalatória e aumento da dose de CI.

Finalmente, o presente estudo mostrou que a quantificação da FeNO foi melhor que a espirometria e a presença de sintomas para avaliar asmáticos atópicos, e os resultados sugerem que asmáticos atópicos parecem estar mais susceptíveis a recaídas que os não-atópicos.

\section{Referências}

1. Baraldi E, Azzolin N, Carra S, Zachello F. Application of exhaled nitric measurement in paediatrics. Eur Respir Rev. 1999;9: 234-40.

2. Deykin AD. Targeting biologic markers in asthma - is exhaled nitric oxide the bull's-eye? N Engl J Med. 2005;352: 2233-5.

3. Beilman G. Exhaled nitric oxide in pathophysiologic states. Chest. 2004;125:11-3.

4. de Jongste JC, Alving K. Gas analysis. Am J Respir Crit Care Med. 2000;162:S23-7.

5. Chiron R, Vachier I, Godard P, Chanez P. La mesure du monoxide d'azote exhalé, un nouvel outil la prise en charge de l'asthme. Presse Med. 2004;33:1451-8.

6. Mattes J, Gravesande KS, Reining U, Alving K, Ihorst G, Henschen $M$, et al. NO in exhaled air is correlated with markers of eosinophilic airway inflammation in corticosteroid- dependent childhood asthma. Eur Respir J. 1999;13:1391-5.

7. de Jongste JC. Surrogate markers of airway inflammation: inflammometry in paediatric respiratory medicine. Paediatr Respir Rev. 2000;1:354-60.

8. Baraldi E, de Jongste JC; ERS/ATS Task Force. Measurement of exhaled nitric oxide in children. Eur Respir J. 2002;20:223-37.

9. Piacentini GL, Bodini A, Costella S, Vicentini L, Mazzi P, Speradio $S$, et al. Exhaled nitric oxide and eosinophil markers of inflammation in asthmatic children. Eur Respir J. 1999;13: 1386-90.

10. Beck-Ripp J, Griese M, Arenz S, Korig C, Pasqualoni B, Buffer P. Changes of exhaled nitric oxide during steroid treatment of childhood asthma. Eur Respir J. 2002;19:1015-9.

11. Global Initiative for Asthma: Global Strategy for Management and Prevention. NHLBI \WHO workshop report. Bethesda: NIH; 2002. p. 1-175.

12. American Thoracic Society Documents. ATS/ERS recommendations for standardized procedures for the on-line and off-line measurement of exhaled lower respiratory nitric oxide and nasal nitric oxide. Am J Respir Crit Care Med. 2005;171:912-30.

13. Steerenberg PA, van Amsterdam JG. Measurement of exhaled nitric oxide. Methods Mol Biol. 2004;279:45-68.

14. Dupont LJ, Demedts MG, Verleden GM. Prospective evaluation of the validity of exhaled nitric oxide for the diagnosis of asthma. Chest. 2003;123:751-6.

15. Franklin PJ, Taplin R, Stick SM. A community study of exhaled nitric oxide in healthy children. Am J Respir Crit Care Med. 1999;159:69-73.

16. Baraldi E, Azzolin NM, Cracco A, Zacchello F. Reference values of exhaled nitric oxide for healthy children 6-15 years old. Pediatr Pneumol. 1999;27:54-8.

17. Nordvall SL, Janson C, Kalm-Stephens P, Foucard T, Toren K, Alving $\mathrm{K}$. Exhaled nitric oxide in a population-based study of asthma and allergy in schoolchildren. Allergy. 2005;60:469-75.

18. Arttlich A, Busch T, Lewandowski K, Jonas S, Gortner L, Falke KJ. Childhood asthma: exhaled nitric oxide in relation to clinical symptoms. Eur Respir J. 1999;13:1396-401.

19. Mahut B, Delacourt C, Zerah-Lancner F, de Blic J, Harf A, Delclaux $C$. Increase in alveolar nitric oxide in the presence of symptoms in childhood asthma. Chest. 2004;125:1012-8.

20. Leuppi JD, Downs SH, Downie SR, Marks GB, Salome CM. Exhaled nitric oxide levels in atopic children: relation to specific allergic sensitisation, AHR, and respiratory symptoms. Thorax. 2002;57:518-23.

21. Brussee JE, Smith HA, Kerkhof M, Koopman LP, Wijga AH, Postma DS, et al. Exhaled nitric oxide in 4-year-old children: relationship with asthma and atopy. Eur Respir J. 2005;25: 455-61.

22. Silvestri $M$, Sabatini $F$, Spallarossa D, Fregonese $L$, Battistini $E$, Biraghi MG, et al. Exhaled nitric oxide levels in non-allergic and allergic mono- or polysensitised children with asthma. Thorax. 2001;56:857-62.

23. Buchvald $\mathrm{F}$, Eiberg $\mathrm{H}$, Bisgaard $\mathrm{H}$. Heterogeneity of FeNO response to inhaled steroid in asthmatic children. Clin Exp Allergy. 2003;33:1735-40.

24. Smith AD, Cowan JO, Brasset KP, Herbison GP, Taylor DR. Use of exhaled nitric oxide measurements to guide treatment in chronic asthma. N Engl J Med. 2005;352:2163-73.

25. Pijnenburg MW, Bakker EM, Lever S, Hop WC, de Jongste JC. High fractional concentration of nitric oxide in exhaled air despite steroid treatment in asthmatic children. Clin Exp Allergy. $2005 ; 35: 920-5$

\section{Correspondência:}

Paulo A. M. Camargos

Departamento de Pediatria da Faculdade de Medicina

Universidade Federal de Minas Gerais

Av. Alfredo Balena, 190, sala 4061

CEP 30130-100 - Belo Horizonte, MG

Tel.: (31) 3248.9773 / Fax: (31) 3248.9664

E-mail: pcamargs@medicina.ufmg.br 